\begin{tabular}{|c|c|}
\hline Title & Characteristics of magnetic island formation due to resistive interchange instability in helical plasma \\
\hline Author(s) & Ueda, R.; W atanabe, K. Y.; Matsumoto, Y.; Itagaki, M.; Sato, M.; Oikawa, S. \\
\hline Citation & $\begin{array}{l}\text { Physics of Plasmas, 21(5), 052502-1-052502-8 } \\
\text { https://doi.org/10.1063/1.4875340 }\end{array}$ \\
\hline Issue Date & 201405 \\
\hline Doc URL & http:/hdl.handle.net/2115/57111 \\
\hline Rights & $\begin{array}{l}\text { Copyright } 2014 \text { A merican Institute of Phy sics. This article may be downloaded for personal use only. Any other use } \\
\text { requires prior permission of the author and the A merican Institute of Physics. The following article appeared in Physics } \\
\text { of Plasmas vol.21 and may be found at } h t t p: / / d x \text {.doi.org } / 10.1063 / 1.4875340\end{array}$ \\
\hline Type & article \\
\hline File Information & 1.4875340.pdf \\
\hline
\end{tabular}

Instructions for use 


\section{AIP $\left.\right|_{\text {Physics of }}$ \\ Plasmas}

\section{Characteristics of magnetic island formation due to resistive interchange instability in helical plasma}

R. Ueda, K. Y. Watanabe, Y. Matsumoto, M. Itagaki, M. Sato, and S. Oikawa

Citation: Physics of Plasmas (1994-present) 21, 052502 (2014); doi: 10.1063/1.4875340

View online: http://dx.doi.org/10.1063/1.4875340

View Table of Contents: http://scitation.aip.org/content/aip/journal/pop/21/5?ver=pdfcov

Published by the AIP Publishing

\section{Articles you may be interested in}

Three dimensional instabilities of an electron scale current sheet in collisionless magnetic reconnection

Phys. Plasmas 21, 062116 (2014); 10.1063/1.4885636

Nonlinear stability of magnetic islands in a rotating helical plasma

Phys. Plasmas 19, 122510 (2012); 10.1063/1.4773041

Hyper-resistivity and electron thermal conductivity due to destroyed magnetic surfaces in axisymmetric plasma equilibria

Phys. Plasmas 19, 062502 (2012); 10.1063/1.4728080

Numerical simulations of separatrix instabilities in collisionless magnetic reconnection

Phys. Plasmas 19, 042110 (2012); 10.1063/1.3698621

Measurements of the momentum and current transport from tearing instability in the Madison Symmetric Torus reversed-field pincha)

Phys. Plasmas 16, 055903 (2009); 10.1063/1.3090325

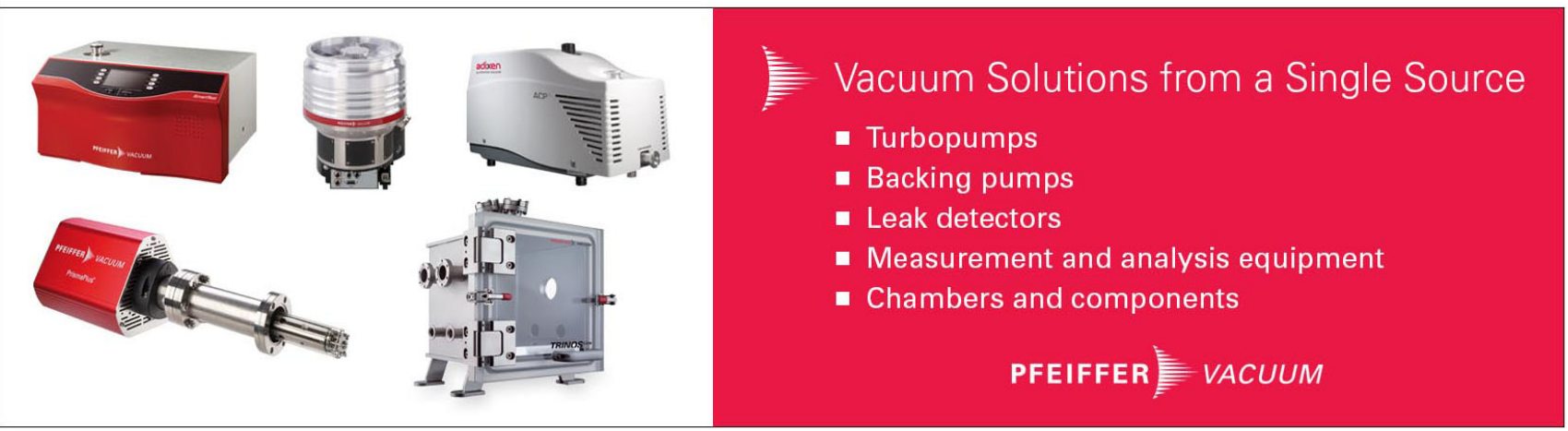




\title{
Characteristics of magnetic island formation due to resistive interchange instability in helical plasma
}

\author{
R. Ueda, ${ }^{1}$ K. Y. Watanabe, ${ }^{2}$ Y. Matsumoto, ${ }^{1}$ M. Itagaki, ${ }^{1}$ M. Sato, ${ }^{2}$ and S. Oikawa ${ }^{1}$ \\ ${ }^{1}$ Graduate School of Hokkaido University, Sapporo 060-8628, Japan \\ ${ }^{2}$ National Institute for Fusion Science, 322-6 Oroshi-cho, Toki 509-5292, Japan
}

(Received 22 January 2014; accepted 15 March 2014; published online 8 May 2014)

Focusing attention on the magnetic island formation, we investigate the characteristics of the resistive interchange magnetohydrodynamics instabilities, which would limit a high beta operational regime in helical type fusion reactors. An introduction of a new index, i.e., the ratio of the magnetic fluctuation level to the radial displacement, enables us to make a systematic analysis on the magnetic island formation in the large helical device-like plasmas during the linear growth phase; (i) the interchange instability with the second largest growth rate makes the magnetic island larger than that with the largest growth rate when the amplitude of the radial displacement in both cases is almost the same as each other; (ii) applied to a typical tearing instability, the index is smaller than that for the interchange instability with the second largest growth rate. (C) 2014 AIP Publishing LLC. [http://dx.doi.org/10.1063/1.4875340]

\section{INTRODUCTION}

The Large Helical Device (LHD) is the largest helicaltype plasma experimental device, which has achieved a plasma with a high beta $(\beta)$ value that is equivalent to a fusion reactor. The $\beta$ value, the ratio of the plasma pressure to the magnetic pressure, is an indicator to evaluate the economic efficiency. Since helical-type devices do not require a toroidal current intrinsically, they have an advantage that the currentdriven instability does not appear, and are suitable for steady state operation. However, the pressure-driven instability such as the interchange instability appears in helical-type devices. For high $\beta$ plasmas in the LHD, since the magnetic hill exists in the peripheral region, there is always a possibility that the resistive interchange instability appears. It is known that the resistive interchange instability causes a degradation of plasma confinement properties. ${ }^{1}$ Therefore, it is one of the crucial issues for the realization of the fusion reactor to study the characteristics of the resistive interchange instability.

In the LHD experiments, magnetohydrodynamics (MHD) activities have been observed by use of magnetic probes, electron cyclotron emission (ECE), and soft X-ray (SX) measurements. ${ }^{2-4}$ With these measurements, we can investigate structures of instabilities, e.g., the toroidal and the poloidal structure, the radial profile of the radial displacements and the perturbed temperature. According to the measured magnetic fluctuation resonated with the low-ordered rational surface, the magnetic fluctuation level increases with the decrease in the magnetic Reynolds number (the $S$ number) and the increase in the $\beta$ value. ${ }^{2}$ The coherent magnetic fluctuation observed outside the plasma implies an existence of the perturbed current resonated with the rational surface. It is considered that the perturbed currents due to MHD instabilities induce the magnetic island formation. Moreover, due to the presence of the fluctuations in the marginally unstable plasma, an apparent degradation in the electron temperature gradients and in the confinement performance is observed. ${ }^{1}$
On the other hand, the characteristics of the resistive interchange instability have been studied from the viewpoint of the dependence of the linear growth rate and the width of the radial perturbed profile on the $\beta$ and the $S$ number, and from the aspect of their nonlinear saturation behavior as well. An early theoretical work on the resistive interchange instability was made by Furth et al. ${ }^{5}$ They clarified that the linear growth rate and the width of the radial profile are proportional to $S^{-1 / 3}{ }^{5}$ Other numerical studies also clarified the characteristics of the resistive interchange instability in the linear growth phase. For example, Ichiguchi et al. ${ }^{6}$ carried out the linear analysis for the heliotron plasma. They showed that the linear growth rate is proportional to $S^{-1 / 3}$ in the low $\beta$ plasma where the ideal interchange instability is negligible. Furthermore, it was shown that the dependence of the growth rate on the $S$ number deviates from $S^{-1 / 3}$ in high $\beta$ plasma where the ideal interchange instability is definite. ${ }^{6}$ There are also many simulation studies about the effect of the resistive interchange instability in the nonlinear saturation phase on the plasma confinement property. ${ }^{7-11}$ The characteristics of the perturbed current due to the resistive interchange instability and the consequent magnetic island have been reported, although the reports are for the limited range of the $\beta$ value and the $S$ number. However, it is increasing for the demand of the systematical prediction of the characteristics of the resistive interchange instability, e.g., the magnetic island formation property, to interpret the LHD experiments results. ${ }^{12,13}$ The reasons for this are as follows. The ranges of the $\beta$ and the $S$ numbers covered in the LHD experiments have become wider, and the measurement accuracy of the perturbation profile has been improved. These improvements enable us to measure systematically the plasma pressure profile with crucial effect on the instability and the rotational transform profile, and enable us to expand the experimental database of the appearance domain for the resistive interchange instability. 
In this study, we propose a new index which is made up of a combination of the magnetic fluctuation level and the amplitude of the radial displacement. The index enables us to investigate the behavior of the magnetic island formation even in the linear growth phase. This investigation has been systematically performed against a configuration to model the LHD plasma in the linear growth phase and also in a wide range of the $\beta$ value and the $S$ number. We focus on the $m / n=1 / 1$ mode instability resonated with the peripheral rational surface, because such low-ordered mode is observed in a wide range of $\beta$ value in the LHD experiments. Here, $m$ and $n$ are the poloidal and the toroidal mode numbers, respectively. Our analysis is carried out based on an eigenvalue problem approach. We investigate not only the characteristics of the first eigenfunction but also the characteristics of the second and the third eigenfunction. The first, the second, and the third eigenfunction correspond to the largest, the second largest, and the third largest growth rates, respectively.

\section{NUMERICAL MODELS}

\section{A. Model equations}

The following linearized equations are employed in this study:

$$
\begin{gathered}
\frac{\partial \tilde{\psi}}{\partial \tau}=\nabla_{\|} \tilde{\phi}+\bar{\eta} \nabla_{\perp}^{2} \tilde{\psi} \\
\frac{\partial}{\partial \tau} \nabla_{\perp}^{2} \tilde{\phi}=\nabla_{\|} \nabla_{\perp}^{2} \tilde{\psi}+\nabla J_{0} \times \nabla \tilde{\psi} \cdot \boldsymbol{e}_{\zeta}+\nabla \Omega \times \nabla \tilde{p} \cdot \boldsymbol{e}_{\zeta}
\end{gathered}
$$

and

$$
\frac{\partial \tilde{p}}{\partial \tau}=\nabla \tilde{\phi} \times \nabla p_{0} \cdot \boldsymbol{e}_{\zeta} .
$$

Equations (1)-(3) are based on the reduced MHD equations $^{14}$ described in cylindrical coordinates $(\rho, \theta, \zeta)$. The variables $\tilde{\psi}, \tilde{\phi}$, and $\tilde{p}$ are the perturbed components of the poloidal flux, the stream function, the plasma pressure normalized by $\epsilon a B_{0}, \epsilon a V_{A}$, and $\epsilon B_{0}^{2} \mu_{0}$, respectively. $\bar{\eta} \equiv \eta /$ $\left(\mu_{0} \epsilon a V_{A}\right)$ denotes the normalized resistivity. Time and radius are also normalized as $\tau \equiv t /\left(a /\left(\epsilon V_{A}\right)\right)$ and $\rho \equiv r / a$, respectively. $\epsilon$ is the inversed plasma aspect ratio ( $\equiv a / R_{0}$ ). Here, $a$ and $R_{0}$ are the plasma minor radius and the major radius, respectively. $J_{0}$ and $p_{0}$ are the normalized equilibrium toroidal current and the normalized equilibrium pressure. Here, $V_{A}$ is the Alfvén velocity. The magnetic Reynolds number ( $S$ number) is defined as $S=\bar{\eta}^{-1}$. The operators $\nabla_{\|}$and $\nabla_{\perp}$ are defined as $\nabla_{\|} \equiv\left(\boldsymbol{B}_{0} / B_{0}\right) \cdot \nabla$ and $\nabla_{\perp}^{2} \equiv \nabla^{2}-\partial^{2} / \partial \zeta^{2}$. The contribution of the magnetic curvature is expressed using $\Omega$ in Eq. (2), which is given by

$$
\Omega^{\prime}=\frac{N \epsilon}{\ell} \frac{1}{\rho^{2}}\left(\rho^{4} \imath\right)^{\prime},
$$

with ' $\equiv d / d \rho . N$ is the pitch number and $\ell$ is the pole number. In the LHD, $N=10, \ell=2$, and $\epsilon=0.6 / 3$.6. The symbol $l$ denotes the rotational transform. With a Fourier-Laplace transformation, the perturbed components $(\tilde{\psi}, \tilde{\phi}, \tilde{p})$ are expressed as $(\hat{\psi}, \hat{\phi}, \hat{p}) \exp (i(m \theta-n \zeta)-i \omega \tau)$. We assume that both $\tilde{\psi}$ and $\tilde{p}$ have only real part, while both $\tilde{\phi}$ and $\omega$ have only imaginary part. Substituting these expressions to Eqs. (1)-(3), we obtain

$$
\begin{gathered}
\gamma \hat{\psi}=-k_{\|} \hat{\phi}+\bar{\eta} \hat{\nabla}_{\perp}^{2} \hat{\psi}, \\
\gamma \hat{\nabla}_{\perp}^{2} \hat{\phi}=k_{\|} \hat{\nabla}_{\perp}^{2} \hat{\psi}+\frac{m}{\rho}\left(\Omega^{\prime} \hat{p}+J_{0}^{\prime} \hat{\psi}\right),
\end{gathered}
$$

and

$$
\gamma \hat{p}=\frac{m}{\rho} p_{0}^{\prime} \hat{\phi}
$$

where

$$
\begin{gathered}
k_{\|}=m \imath-n \\
\hat{\nabla}_{\perp}^{2} \equiv \frac{1}{\rho} \frac{d}{d \rho} \rho \frac{d}{d \rho}-\frac{m^{2}}{\rho^{2}} .
\end{gathered}
$$

It is noted that $\omega$ has been replaced by $i \gamma$, where $\gamma$ is the linear growth rate normalized by the poloidal Alfvén time. Equations (5)-(7) are solved as an eigenvalue problem with $\gamma$ being the eigenvalue.

\section{B. Numerical methods}

Assuming the LHD plasmas to be current-less in our interchange instability analysis, we ignore the equilibrium current $J_{0}$. The boundary conditions

$$
\hat{\psi}=0 \text { and } \hat{\phi}=0
$$

are set on the position $\rho=0$ and $\rho=1$. Additionally, we give the boundary condition $\hat{\psi}^{\prime}(\rho=1)=1$ in order to remove a trivial solution. Under the boundary conditions except for $\hat{\psi}(\rho=1)=0$, we solve Eqs. (5)-(7) with given initial approximation $\gamma$, and iteratively update $\gamma$ until the boundary conditions on $\rho=1$ are satisfied. The eigenfunction of $\hat{p}$ is determined by Eq. (7). The derivative with respect to $\rho$ is obtained using the second-order accurate central-difference scheme. The interval $0 \leq \rho \leq 1$ is divided equidistantly into 5000 grids. We investigate the characteristics of the instabilities from the radial perturbation profiles of the poloidal flux, the current density, and the radial displacement. Using the radial perturbation profiles of $\hat{\psi}, \hat{\phi}$, and $\hat{p}$, we can express the radial profile of the perturbed current density and the radial displacement as

$$
\hat{j}=\hat{\nabla}_{\perp}^{2} \hat{\psi}
$$

and

$$
\hat{\xi}_{r}=-\frac{m \hat{\phi}}{\gamma \rho},
$$

respectively. 


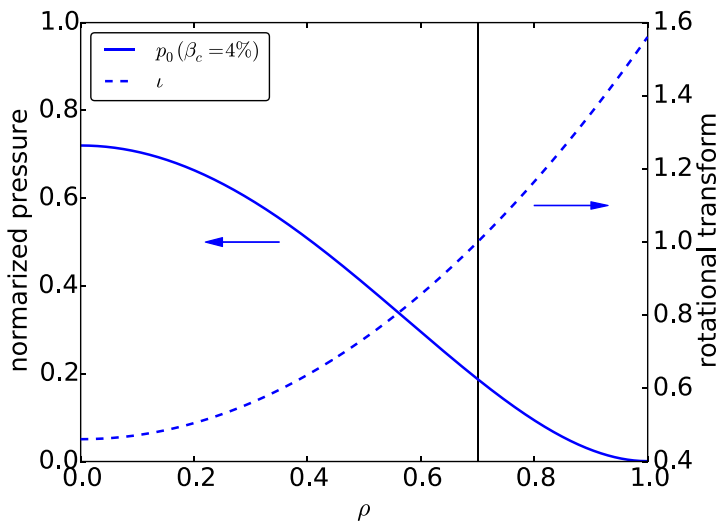

FIG. 1. Equilibrium profile of the pressure (solid line) and the rotational transform (dashed line). Vertical line denotes the position of $l=1$ rational surface.

\section{Equilibrium profile}

Typical equilibrium profiles

$$
p_{0}=\frac{1}{2 \epsilon^{2}}\left[\left(1-p_{a}\right)\left(1-\rho^{2}\right)^{2}+p_{a}\right] \beta_{c},
$$

and

$$
\iota=-\frac{1}{\rho} \frac{d \psi_{0}}{d \rho}=l_{c}+\left(l_{w}-l_{c}\right) \rho^{2},
$$

are used for the interchange instability analysis. We assume $p_{a}=10^{-3}, l_{c}=0.461$, and $\imath_{w}=1.561$. The symbol $\beta_{c}$ here denotes the central beta, the value of which is changed variously for parameter survey. Figure 1 shows the rotational transform and the pressure profile for $\beta_{c}=4 \%$. The $(m, n)=(1,1)$ rational surface is located at $\rho=0.7$.

\section{CHARACTERISTICS OF RESISTIVE INTERCHANGE INSTABILITY}

\section{A. Radial structure}

In the plasmas with a finite resistivity, the reconnection of the magnetic lines can occur, so that the magnetic islands can be formed. Focusing attention on the magnetic island formation, we investigate the characteristics of the resistive interchange instability. Figure 2 shows the radial profile of (a) the perturbed poloidal flux, (b) the perturbed current density, and (c) the radial displacement for the first eigenfunction of the resistive interchange instability with $S=10^{4}$, $\beta_{c}=2 \%$. The vertical solid line denotes the location of the resonated rational surface $\imath=1$. The normalized growth rate is $\gamma=2.64 \times 10^{-2}$. The Suydam criterion, the stable limit of the ideal interchange instability, is given by

$$
\frac{1}{4}\left(\frac{d l}{d \rho}\right)^{2}+\frac{1}{\rho^{2}} \frac{d p_{0}}{d \rho} \frac{d \Omega}{d \rho}<0 .
$$

Using this criterion, we obtain the stability limit of the ideal interchange instability as $\beta_{c}=2.3 \%$. Since the calculation condition $\beta_{c}=2 \%$ of Fig. 2 is below the stability limit, the radial profiles represent the typical structures of the resistive interchange instability. It can be seen from Fig. 2(a) that although the perturbed poloidal flux profile has almost the odd structure with respect to the rational surface, the $\hat{\psi}$ value on the rational surface is not exactly zero. The magnetic field is expressed as

$$
\boldsymbol{B}=\boldsymbol{e}_{\zeta} \times \nabla \psi+B_{0} \boldsymbol{e}_{\zeta}
$$

Due to the non-zero $\hat{\psi}$, we know $\hat{B}_{r} \neq 0$ on the resonant rational surface, which leads to the magnetic island formation. We can see from Fig. 2(b) that the perturbed current in radial profile has the opposite directions with respect to the rational surface. The radial displacement shown in Fig. 2(c) has almost the even structure, which means the displacements are the same direction in the vicinity of the rational surface. Next, we describe the characteristics of the eigenfunction with growth rate smaller than the first eigenfunction, although they have the same poloidal and toroidal numbers as the first eigenfunction. For the resistive interchange instability, the growth rates of the second and the third eigenfunctions are the same order as the first eigenfunction, which will be shown in Sec. III B. Figures 3 and 4 show the radial profile of the second and the third eigenfunctions, respectively. Figures 3 and 4 as well as Fig. 2 show (a) the perturbed poloidal flux, (b) the perturbed current density, and (c) the radial displacement. These results are calculated for the same plasma that is shown in Fig. 2. The growth rate of the second and the third eigenfunctions are $\gamma=1.67 \times 10^{-2}$ and $\gamma=8.09 \times 10^{-3}$, respectively. It can be seen from Fig. 3(a) that the radial profile of the perturbed poloidal flux is almost the even structure with respect to the rational surface. The $\hat{\psi}$ value on the rational surface is finite, which is quite different from that of the first eigenfunction. The radial perturbed current density profile of the second eigenfunction shown in Fig. 3(b) has almost the even structure with respect to the rational surface, which is considered to form the magnetic island easily. The directions of the currents inside and outside the rational surface are opposite to that on the surface. The radial displacement profile of the second eigenfunction shown in Fig. 3(c) has almost the odd structure with respect to the rational surface. In the third eigenfunction, the poloidal flux profile shown in Fig. 4(a) represents almost the odd structure with respect to the
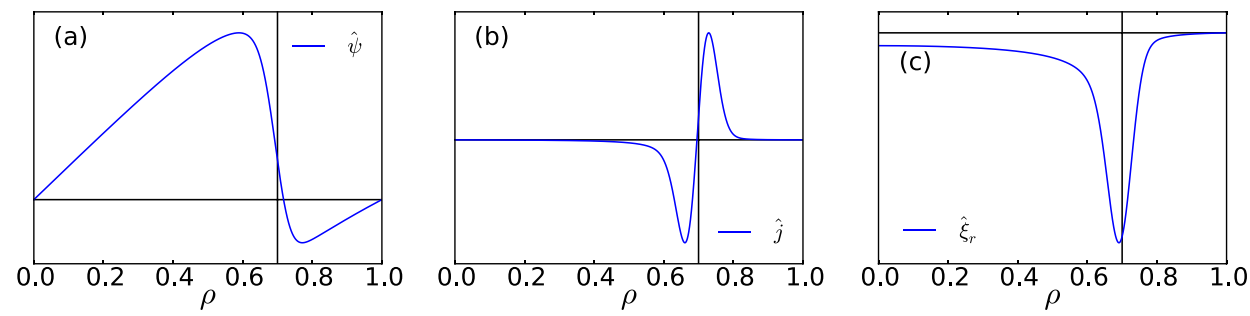

FIG. 2. Radial profile of the eigenfunctions (a) $\hat{\psi}$, (b) $\hat{j}$, and (c) $\hat{\xi}_{r}$ with the largest growth rate. Vertical solid line denotes the position of $l=1$ rational surface. 

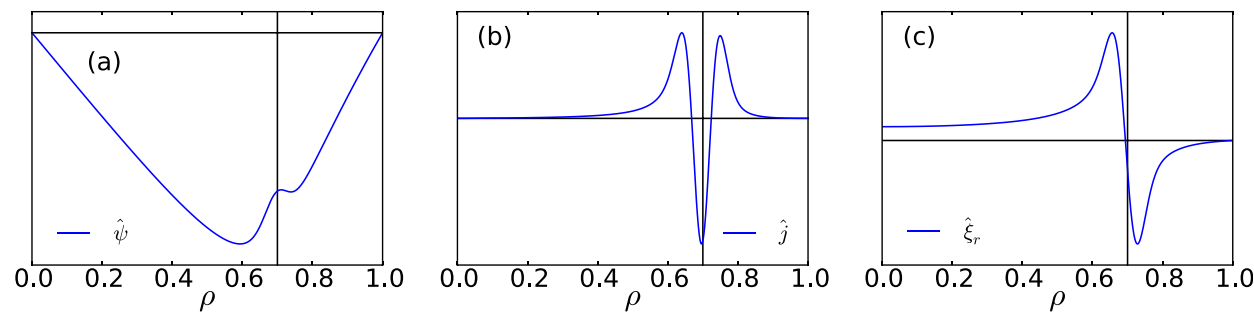

FIG. 3. Radial profile of the eigenfunctions (a) $\hat{\psi}$, (b) $\hat{j}$, and (c) $\hat{\xi}_{r}$ with the second largest growth rate. Vertical solid line denotes the position of $l=1$ rational surface. rational surface. Although this structure looks similar to that of the first eigenfunction, the radial profile of the perturbed current density shown in Fig. 4(b) represents an additional oscillation and different structure from that of the first eigenfunction. The radial displacement profile shown in Fig. 4(c) represents approximately the even structure. The directions of the radial displacements inside and outside the rational surface are opposite to that on the surface. From the radial displacement profiles shown in Figs. 2-4(c), we can find that the first, the second, and the third eigenfunction have one, two, and three extremal points, respectively.

\section{B. Dependence of growth rate and width of radial structure on $\beta$ and magnetic Reynolds number}

It is well known that the plasma pressure and the $S$ number affect the linear growth rate and the width of the radial perturbation structure. ${ }^{1}$ In this section, we investigate these effects for the first, the second, and the third eigenfunctions. Figure 5 shows the linear growth rates of each eigenfunction as a function of the $S$ number for various $\beta$ plasmas with $2 \%<\beta_{c}<5 \%$. For low $\beta$ value and small $S$ number, the linear growth rates of the second and the third eigenfunctions are the same order as those of the first eigenfunction. For high $\beta$ and large $S$ number, however, the growth rates of the first eigenfunction are much larger than those of the second and the third eigenfunctions. It is well known that the growth rate depends on the $S$ number as $\gamma \propto S^{-1 / 35}$. We can see from Fig. 5 that the dependence of $\gamma$ on $S$ is close to $\gamma$ $\propto S^{-1 / 3}$ in low $\beta$. The above dependence implies that not only the first eigenfunction but also the second and the third eigenfunctions have the characteristics of the resistive interchange instability. For the $\beta_{c}>3 \%$ plasmas, the dependence deviates from $\gamma \propto S^{-1 / 3}$. For high $\beta$ regime, in particular, the $S$-dependence disappears. This is because the ideal interchange instability appears over the Suydam limit, i.e., $\beta_{c}=2.3 \%$ for the present equilibrium profile, and becomes gradually dominant with the increase in the $\beta$ value. The similar conclusion is already shown by Ichiguchi et al. ${ }^{6}$ On the contrary, the second and the third eigenfunctions keep the characteristics of the resistive interchange instability, i.e., $\gamma \propto S^{-1 / 3}$ even in high $\beta$ regime. The reason for the clearly appearance of such characteristics is that the growth rates of the second and third eigenfunctions in the ideal interchange instability are much smaller than that of the first eigenfunction as shown in the Appendix. Next, we show in Fig. 6 that the dependence of the width of the radial displacement profile on the $S$ number in the first, the second, and third eigenfunctions. Here, the radial width is evaluated from the FWHM (full width at the half maximum) of the radial displacement profile. Since the second and the third eigenfunctions have multiple extremal points, the FWHM is evaluated for the maximum value of the $\left|\hat{\xi}_{r}\right|$. It can be seen from Fig. 6 that the first, the second, and the third eigenfunctions represent almost the same $S$-dependence of the FWHM, i.e., FWHM $\propto S^{-1 / 3}$ in low $\beta$ values. The $S$-dependence deviates from FWHM $\propto S^{-1 / 3}$ in high $\beta$ regime. This is because the ideal interchange instability becomes dominant as well as the $S$-dependence of the growth rate. For the second and the third eigenfunctions, the deviation from the $S$-dependence of the FWHM is not seen even in large $S$ number. Also, the $S$-dependence differences of the FWHM among the first, the second, and the third eigenfunctions are not significantly recognized in low $\beta$ regime and also in a wide range of the $S$ number. However, we should note that because the second eigenfunction has the two peak values, there is a possibility that the sum of the FWHMs for each peak is larger than the FWHM of the first eigenfunction. Then, the radial region influenced by the second eigenfunction can be wider than that by the first eigenfunction. Similar notices can also be given for the third eigenfunction.

\section{Introduction of an index on the magnetic island formation}

To investigate the characteristics of the magnetic island formation in the linear growth phase of the interchange instability, we focus on the ratio of the magnetic island width to the radial displacement. In this section, the first and the second eigenfunctions are analyzed as examples of an odd and an even structure. Using the normalized poloidal flux and the shear parameter $l^{\prime}$ on the rational surface, we can express the magnetic island width normalized by the minor radius as
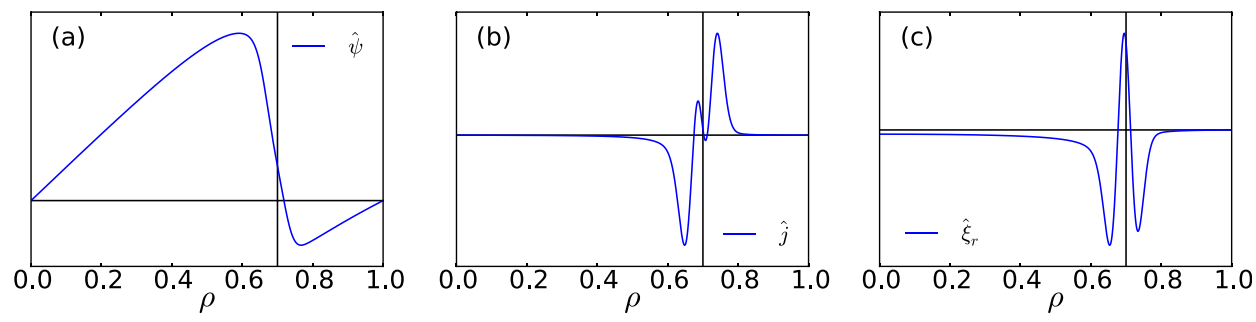

FIG. 4. Radial profile of the eigenfunctions (a) $\hat{\psi}$, (b) $\hat{j}$, and (c) $\hat{\xi}_{r}$ with the third largest growth rate. Vertical solid line denotes the position of $l=1$ rational surface. 

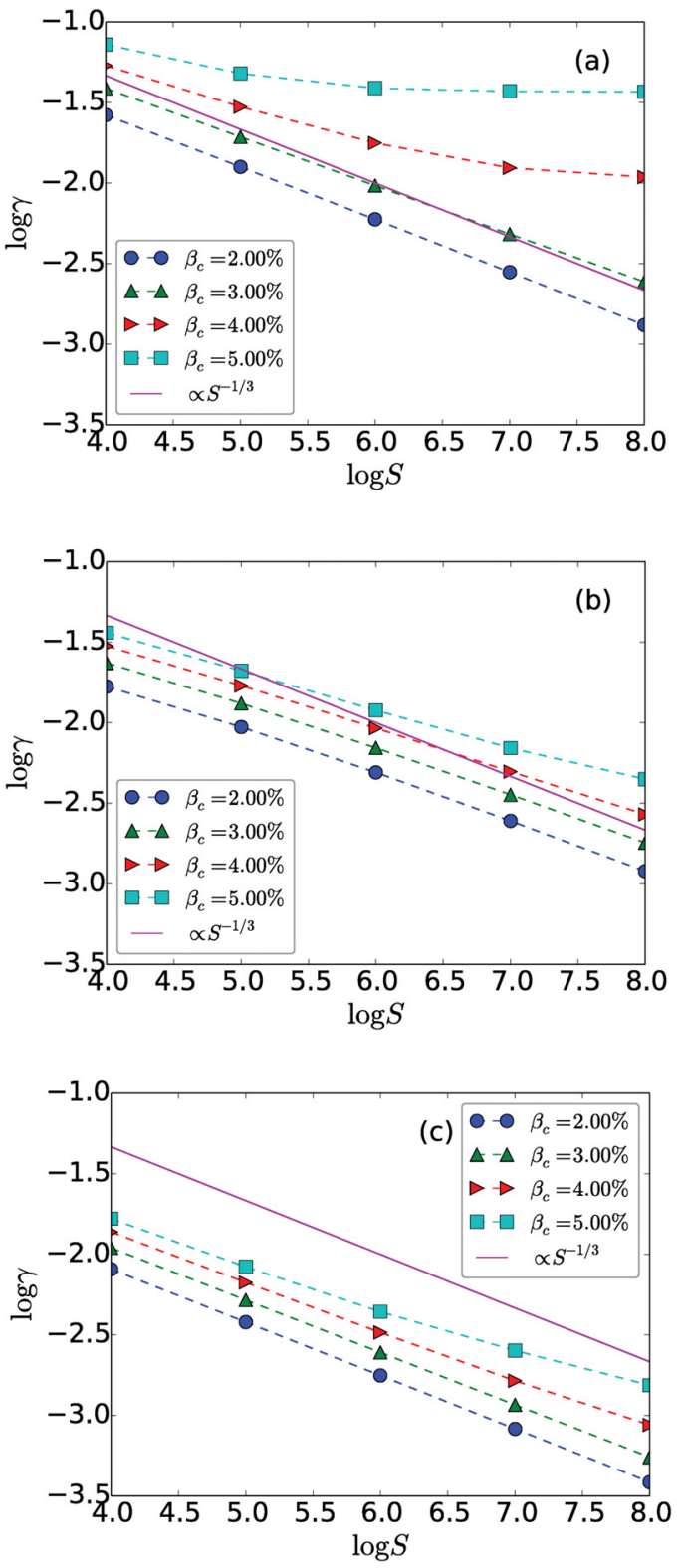

FIG. 5. Linear growth rates of the eigenfunctions with (a) the largest, (b) the second largest, and (c) the third largest growth rates for various $\beta$ plasmas as function of $S$. Solid line denotes $\gamma \propto S^{-1 / 3}$.

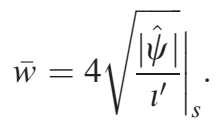

Here, subscript $s$ denotes the rational surface. We cannot directly evaluate the magnetic island width by Eq. (17), since the amplitude of $\hat{\psi}$ on the rational surface cannot be obtained in the linear analysis. On the other hand, we can see $\hat{\psi}_{s} \propto \bar{w}^{2}$ from Eq. (17) and $\hat{\phi} \propto \hat{\xi}_{r}$ from Eq. (12), respectively. Since the ratio of $\hat{\psi}$ to $\hat{\phi}$ is kept in the linear growth phase, the ratio $\bar{w}^{2} / \hat{\xi}_{r}$ could be a good index to characterize the magnetic island formation during the linear growth phase. In the index, we take the maximum values of $\left|\hat{\xi}_{r}\right|$ as the reference $\hat{\xi}_{r}$, which is near the rational surface as shown in Sec. III A. Finally, a new index to characterize the magnetic island formation is defined as
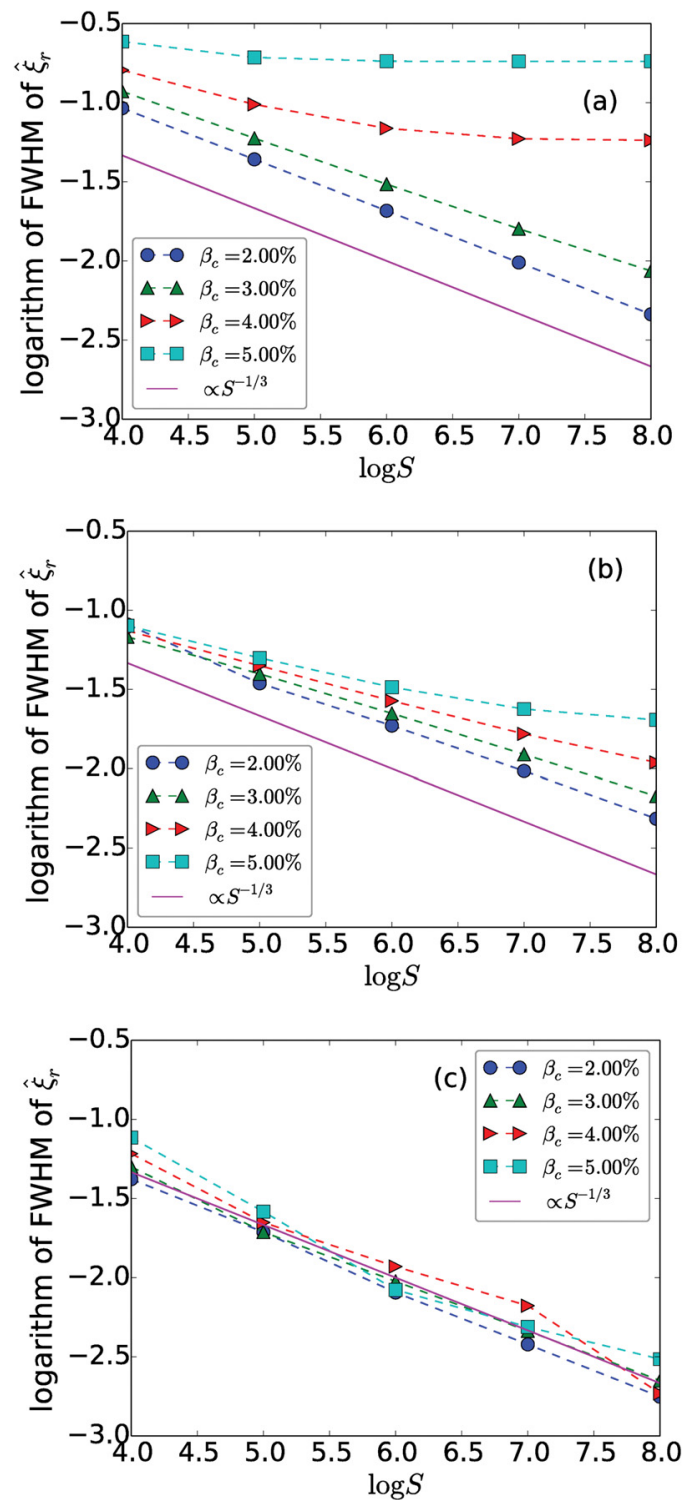

FIG. 6. Linear FWHMs of $\hat{\xi}_{r}$ with (a) the largest, (b) the second largest, and (c) the third largest growth rates for various $\beta$ plasmas as a function of $S$. Solid line denotes FWHM $\propto S^{-1 / 3}$.

$$
I_{\xi} \equiv \frac{w^{2}}{\xi_{r, \text { peak }} a}=\frac{16 \gamma}{m}\left(\frac{|\hat{\psi}|}{\iota^{\prime}}\right)_{s}\left(\frac{\rho}{\hat{\phi}}\right)_{\text {peak }} .
$$

Figure 7 shows the variations of $I_{\xi}$ as a function of the $S$ number for the first and the second eigenfunctions. $I_{\xi}$ for the first eigenfunction of the interchange instability $\left(=I_{\xi, I 1}\right)$ is approximately proportional to $S^{-1 / 3}$ in low $\beta$. Meanwhile, in high $\beta$ and large $S$ number, $I_{\xi, I 1}$ is approximately proportional to $S^{-1}$. $I_{\xi}$ for the second eigenfunction of the interchange instability $\left(=I_{\xi, I 2}\right)$ is proportional to $S^{-1 / 3}$, which can be seen in a beta range wider than $I_{\xi, I 1}$. This is because the linear growth rate of the second eigenfunction for the ideal interchange instability is much smaller than that of the first eigenfunction. Also, we can see that $I_{\xi, I 2}$ is larger than $I_{\xi, I 1}$, which shows that the second eigenfunction forms the magnetic islands larger than the first eigenfunction if the amplitudes of the $\left|\hat{\xi}_{r, \text { peak }}\right|$ in both cases are comparable. 

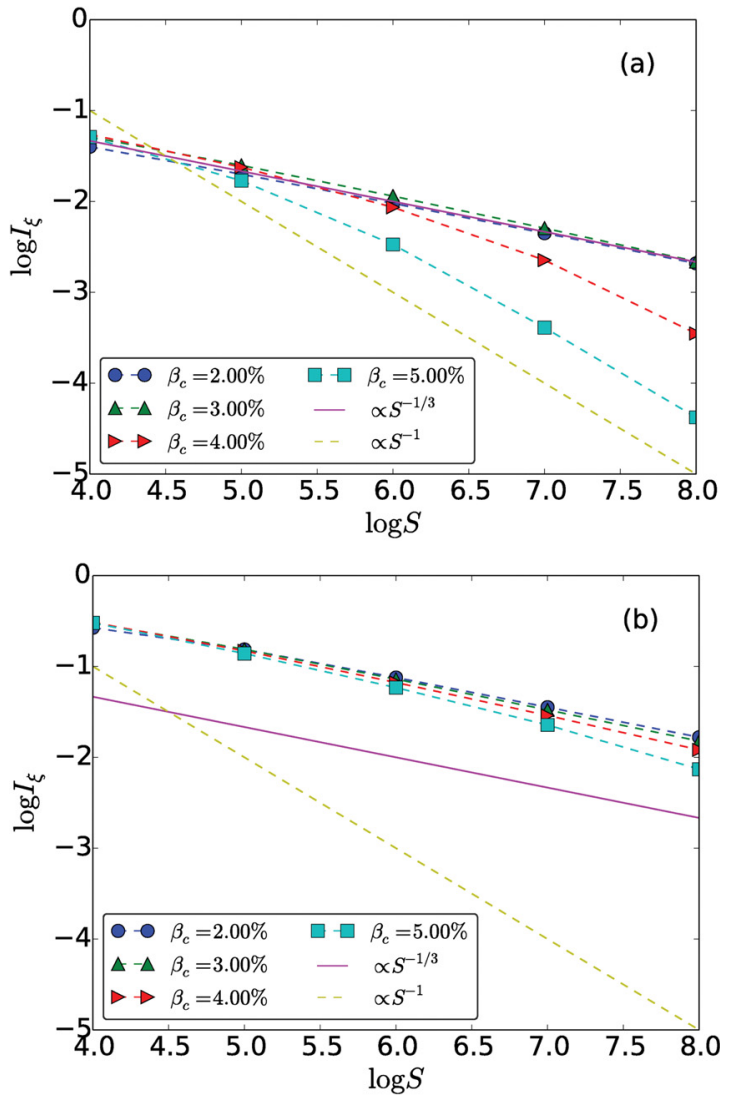

FIG. 7. An index of the magnetic island formation, $I_{\xi}$, of the eigenfunctions with (a) the largest and (b) the second largest growth rates for various $\beta$ plasmas as a function of $S$.

\section{DISCUSSION}

In Sec. III, we studied the aspect of the magnetic island formation due to the resistive interchange instability, and proposed the new index $I_{\xi}$ in Eq. (18). In this section, using the index $I_{\xi}$, we compare the characteristics between the resistive interchange instability and the tearing instability. We also study the characteristics of these instabilities by using another characteristic index on the magnetic island formation. The index is defined as $I_{\psi} \equiv \hat{\psi}_{s} / \hat{\psi}_{\text {peak }}\left(\propto w^{2}\right)$, which can be obtained from only the perturbed poloidal flux. Here, $\hat{\psi}_{\text {peak }}$ denotes the maximum absolute value of the perturbed poloidal flux.

To analyze the characteristics of the magnetic island formed by the tearing instability, the magnetic curvature term $\frac{m}{\rho} \Omega^{\prime} \hat{p}$ in Eq. (6) is ignored. Instead, we include the equilibrium current density term $\frac{m}{\rho} J_{0}^{\prime} \hat{\psi}$ that was once ignored for the interchange instability analysis. Figure 8 shows the radial profiles of (a) the perturbed poloidal flux, (b) the perturbed current density, and (c) the radial displacement of the typical tearing instability. Here, we applied the calculation conditions, $J_{0} \propto\left(1-\rho^{2}\right)^{\nu}, S=10^{4}, \nu=3, l_{c}=0.9, l_{w}=0.225$, and $m / n=2 / 1$. We can see from Fig. 8 (a) that the perturbed poloidal flux of the tearing instability has the clearly finite value on the resonant rational surface. The perturbed current density profile shown in Fig. 8(b) represents the negative maximum value outside the rational surface. On the contrary, the positive current flows inside the rational surface. From the radial displacement profile shown in Fig. 8(c), we observe two peaks which have opposite signs with each other. The peak inside the rational surface is much larger than that outside the surface.

Using the index $I_{\xi}$ proposed in the Sec. III C, we make a comparison between the characteristics on the magnetic island formation due to the resistive interchange instability and those due to the tearing instability. Figure 9 shows $I_{\xi}$ as a function of the $S$ number. It can be seen that $I_{\xi, I 2}$ is the largest index in a wide range of the $\beta$ value and the $S$ number. The index $I_{\xi}$ for the tearing instability $\left(=I_{\xi, T}\right)$ is clearly smaller than $I_{\xi, I 2}$. In small $S$ number, $I_{\xi, T}$ is comparable to $I_{\xi, I 1}$. For high $\beta$ and large $S$ number plasmas with the dominant ideal interchange instability, $I_{\xi, I 1}$ is much small index. These results show that the magnetic island width of the resistive interchange instability is not always smaller than that of the tearing instability if the radial displacements in both cases are comparable. In the LHD experiments, we observed 5\%-40\% radial displacement normalized by the minor radius for the interchange instability. ${ }^{1,13}$ Also, the MHD activities are observed around $10^{5}<S<10^{7} .^{1,2}$ It can be seen from Fig. 7 that $I_{\xi}$ around $10^{5}<S<10^{7}$ is about $10^{-2}$. Therefore, assuming $\xi_{r} / a \sim 5 \%$, it is expected that the magnetic island of $w / a \sim 2 \%$ exists.

Next, using the index $I_{\psi}$, we compare the behavior of the magnetic island formation in the resistive interchange instability with that in the tearing instability. As shown in Fig. 2(a), for the first eigenfunction of the resistive interchange instability, the value $\psi$ on the rational surface is much smaller than the peak $\hat{\psi}$ value. On the contrary, as shown in Fig. 8(a), for the tearing instability, the value $\hat{\psi}$ on the rational surface is about half of the peak $\hat{\psi}$ value. This result coincides with the conventional understanding that the tearing instability forms the magnetic island larger than the resistive interchange instability. Figure 10 shows $I_{\psi}$ as a function of the $S$ number for the resistive interchange instability and the tearing instability. It can be seen that $I_{\psi, T}$ is almost the same as $I_{\psi, I 2}$. The index $I_{\psi, I}$ decreases with increase in the $\beta$ value. The decrease of $I_{\psi, I 1}$ in the $S$ number is large for high $\beta$ plasmas as long as the ideal interchange instability is dominant.
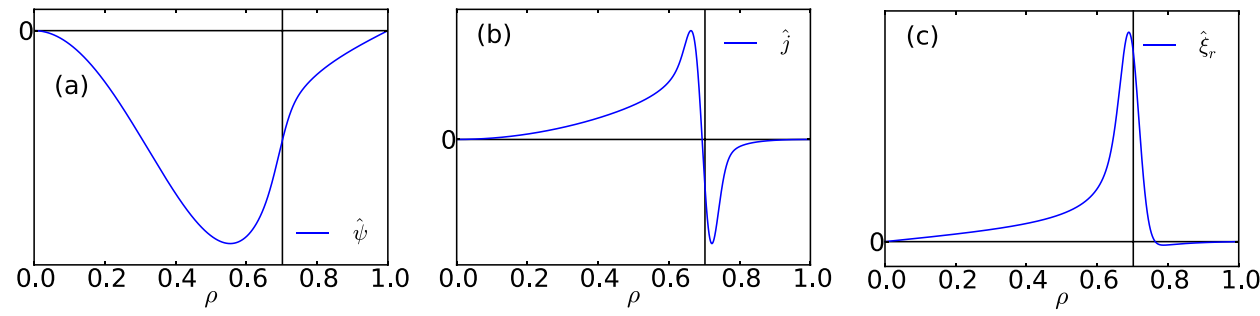

FIG. 8. Radial profiles of the eigenfunctions (a) $\hat{\psi}$, (b) $\hat{j}$, and (c) $\hat{\xi}_{r}$ of the typical tearing instability. 


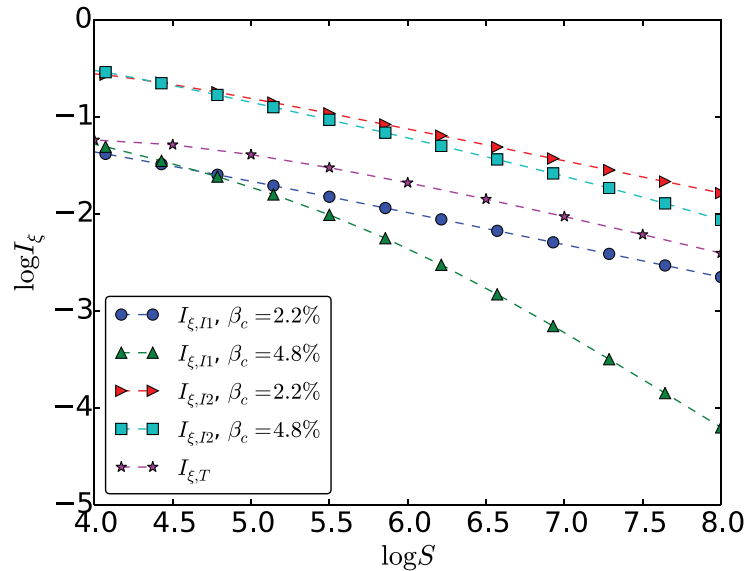

FIG. 9. An index of the magnetic island formation, $I_{\xi}$, of the interchange instabilities and the tearing instability as a function of $S$.

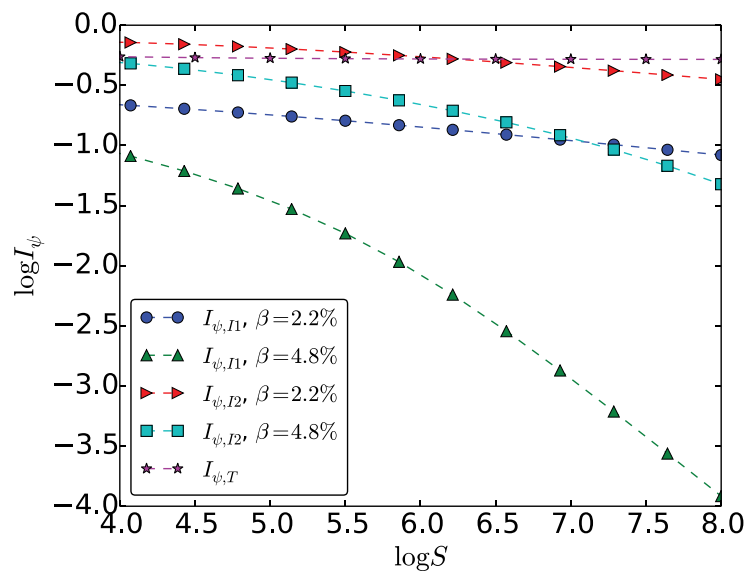

FIG. 10. An index of the magnetic island formation, $I_{\psi}$, of the interchange instabilities and the tearing instability as a function of $S$.

\section{CONCLUSION}

Focusing attention on the magnetic island formation, we numerically investigate the characteristics of the resistive interchange MHD instabilities, which would limit the high $\beta$ operational regime in the helical type fusion reactors. Up to now, there are many early works on the perturbed plasma current and the consequent island formation due to the resistive interchange instabilities. However, typically, the characteristics of the magnetic island formation were studied by the non-linear simulations of the MHD models and the analyzed plasma parameter ranges are limited on the $\beta$ and the magnetic Reynolds number ( $S$ number).

In this paper, we introduce a new index $I_{\xi}$, the ratio of the magnetic fluctuation level to the radial displacement, to characterize the magnetic island formation. Using the index $I_{\xi}$, we systematically analyze the characteristics of the magnetic island formation due to the resistive interchange instabilities during the linear growth phase. The analysis is performed for the $m / n=1 / 1$ interchange instability in the LHD-like configuration, which is observed in the peripheral region of the LHD discharges in a wide range of the $\beta$ value and the $S$ number. Here, $m$ and $n$ are the poloidal and the toroidal mode numbers of the MHD activities, respectively.
For the linear analysis, the reduced MHD equations are solved as the eigenvalue problem. This approach has an advantage that some eigenfunctions with different eigenvalue (growth rate) can be analyzed, that is, the eigenfunctions with the second and the third largest growth rates can be analyzed in addition to that with the largest growth rate. The index $I_{\xi}$ is proportional to the ratio of the square of the normalized magnetic island width to the maximum absolute value of the normalized radial displacement. This index enables us to compare the formed magnetic island width among the instabilities at the same amplitude of the radial displacement.

Using the indexes $I_{\xi}$ for the resistive interchange instability with the largest and the second largest growth rate, we obtain the following results. Here, it should be noted that the second largest growth rate is the same order as the largest growth rate. (i) The formed magnetic island width due to the second fastest growing instability is larger than that due to the fastest growing instability when they have the same amplitude of the radial displacement as each other. (ii) The index $I_{\xi}$ for the fastest growing interchange instability is proportional to $S^{-1 / 3}$ when the ideal interchange instability is negligible, and is proportional to $S^{-1}$ when the ideal instability is definite. On the contrary, $I_{\xi}$ for the second fastest growing interchange instability is proportional to $S^{-1 / 3}$ in a wide range of the $\beta$ value. (iii) The formed magnetic island width due to a typical tearing instability is smaller than that due to the second fastest growing instability of the resistive interchange instability when these instabilities have the same amplitude of the radial displacement as each other. Also, it is shown that the magnetic island width due to the tearing instability is almost the same as that due to the fastest growing resistive interchange instability.

On the contrary, another index of the magnetic island formation $I_{\psi}$, the ratio of the poloidal magnetic flux at the resonant rational surface to that at the maximum absolute value, shows that the tearing instability has the magnetic island width much larger than the fastest growing interchange instability. The magnetic island width due to the tearing instability is almost the same as that due to the second fastest growing interchange instability, which supports the conventional understandings on the magnetic island formation for the tearing instability and the interchange instability.

\section{ACKNOWLEDGMENTS}

This work was performed under the auspices of NIFS Collaboration Research program (NIFS12KNWP002 and NIFS13KLPP031). This work was also supported by the Ministry of Education, Culture, Sports, Science and Technology, Grant-in-Aid for Scientific Research (B), 25289342.

\section{APPENDIX: CHARACTERISTICS OF IDEAL INTERCHANGE INSTABILITY}

The ideal interchange instability of the cylindrical plasma appears when the $\beta$ value exceeds the Suydam limit. For such an ideal unstable plasma, it is difficult to analyze 

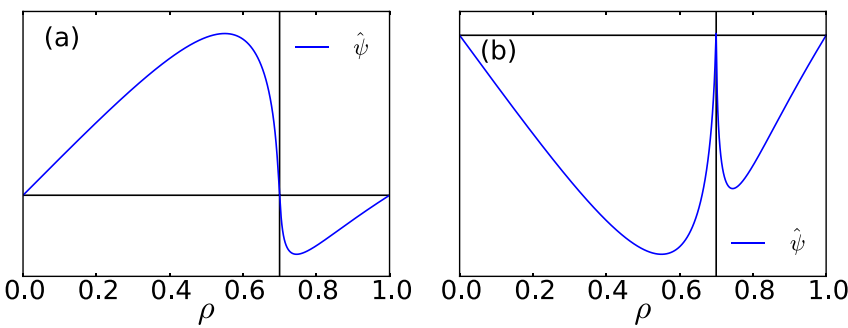

FIG. 11. Radial profiles of an eigenfunction $\hat{\psi}$ of an ideal interchange instability with the largest and the second largest growth rates.

only the resistive interchange instability because the ideal interchange instability appears simultaneously. This is why we must also investigate the characteristics of the ideal interchange instability. The ideal MHD instabilities can be calculated by substituting $\bar{\eta}$ to 0 in Eqs. (5)-(7). Figure 11 shows that the radial perturbation profile of the first and the second eigenfunction of $\hat{\psi}$. The configuration parameters are the exactly same as that in Sec. III. However, the $\beta$ value is given as $\beta_{c}=4.0 \%$ which is much larger than Suydam limit, $\beta_{c}=2.3 \%$. The growth rates of these eigenfunctions are $1.06 \times 10^{-2}, 7.05 \times 10^{-4}$, respectively. It should be noted that the growth rate of the second eigenfunction is much different from that of the first eigenfunction. It can be seen from Fig. 11(a) that the radial perturbation profile of $\hat{\psi}$ in the first eigenfunction is the odd structure with respect to the rational surface. On the other hand, the radial perturbation profile of the second eigenfunction is the even and singular structure with respect to the rational surface. Here, it should be noticed that the $\hat{\psi}$ is substantially zero at the position of the rational surface. Therefore, the X-point or the magnetic islands do not occur on the rational surface. This supports the fact that the magnetic reconnection does not occur in the ideal plasma. Figure 12 shows that the radial perturbation profile of the first and the second eigenfunctions of $\hat{j}$ around the rational surface. We can see that the first eigenfunction of $\hat{j}$ is almost the odd structure and the directions of the perturbed currents are opposite in the vicinity of the rational surface. On the other hand, the radial perturbation profile of the second eigenfunction of $\hat{j}$ is the even structure with

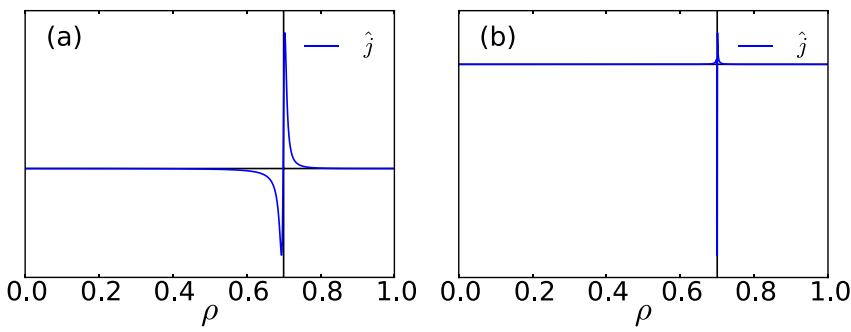

FIG. 12. Radial profiles of an eigenfunction $\hat{j}$ of an ideal interchange instability with the largest and the second largest growth rates.

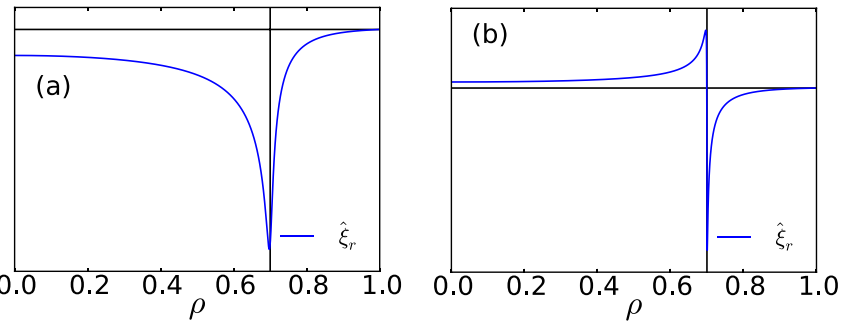

FIG. 13. Radial profiles of an eigenfunction $\hat{\xi}_{r}$ of an ideal interchange instability with the largest and the second largest growth rates.

respect to the rational surface and the singular large current appears on the rational surface. Figure 13 shows the radial perturbation profile of the first and the second eigenfunctions of $\hat{\xi}_{r}$. It can be seen that each eigenfunction has a singular structure on the rational surface. The first eigenfunction has an extremal point and the even structure with respect to the rational surface. On the other hand, the second eigenfunction has two extremal points and the odd structure with respect to the rational surface.

${ }^{1}$ K. Y. Watanabe, S. Masamune, Y. Takemura, H. Funaba, S. Sakakibara, F. Watanabe, K. Tanaka, S. Ohdachi, K. Toi, Y. Narushima, and The LHD Experimental Group, Phys. Plasmas 18, 056119 (2011).

${ }^{2}$ S. Sakakibara, K. Y. Watanabe, Y. Suzuki, Y. Narushima, S. Ohdachi, N. Nakajima, F. Watanabe, L. Garcia, A. Weller, K. Toi, I. Yamada, K. Tanaka, T. Tokuzawa, K. Ida, H. Yamada, A. Komori, O. Motojima, and The LHD Experimental Group, Plasma Phys. Control. Fusion 50, 124014 (2008).

${ }^{3}$ A. Isayama, S. Inagaki, K. Y. Watanabe, Y. Narushima, S. Sakakibara, H. Funaba, K. Ida, Y. Nagayama, H. Yamada, K. Kawahata, A. Komori, O. Motojima, and The LHD Experimental Group, Plasma Phys. Control. Fusion 48, L45 (2006).

${ }^{4}$ F. Watanabe, K. Toi, S. Ohdachi, S. Takagi, K. Y. Watanabe, S. Morita, K. Narihara, K. Tanaka, K. Yamazaki, and The LHD Experimental Group, Plasma Phys. Control. Fusion 48, A201 (2006).

${ }^{5}$ H. P. Furth, J. Killeen, and M. N. Rosenbluth, Phys. Fluids 6, 459 (1963).

${ }^{6}$ K. Ichiguchi, Y. Nakamura, M. Wakatani, N. Yanagi, and S. Morimoto, Nucl. Fusion 29, 2093 (1989).

${ }^{7}$ K. Ichiguchi, N. Nakajima, M. Wakatani, B. A. Carreras, and V. E. Lynch, Nucl. Fusion 43, 1101 (2003).

${ }^{8}$ K. Ichiguchi and B. A. Carreras J. Plasma Fusion Res. Series 6, 589 (2004).

${ }^{9}$ H. Miura, N. Nakajima, T. Hayashi, and M. Okamoto, AIP Conf. Proc. 871, 157 (2006).

${ }^{10}$ J. Varela, K. Y. Watanabe, and S. Ohdachi, Phys. Plasmas 19, 082512 (2012).

${ }^{11}$ B. A. Carreras, V. E. Lynch, H. Zushi, K. Ichiguchi, and M. Wakatani, Phys. Plasmas 5, 3700 (1998).

${ }^{12}$ K. Y. Watanabe, S. Sakakibara, Y. Narushima, H. Funaba, K. Narihara, K. Tanaka, T. Yamaguchi, K. Toi, S. Ohdachi, O. Kaneko, H. Yamada, Y. Suzuki, W. A. Cooper, S. Murakami, N. Nakajima, I. Yamada, K. Kawahata, T. Tokuzawa, A. Komori, and The LHD Experimental Group, Nucl. Fusion 45, 1247 (2005).

${ }^{13}$ K. Y. Watanabe, S. Sakakibara, H. Funaba, S. Ohdachi, K. Tanaka, I. Yamada, L. Garcia, Y. Narushima, C. Michel, H. Yamada, Y. Suzuki, N. Nakajima, K. Narihara, T. Tokuzawa, K. Kawahata, and The LHD Experimental Group, in Proceedings of the Joint Conference of 17th International Toki Conference and 16th International Stella./Heliotron WS, Toki, 2007, 15-19 October 2007, pp. I-13.

${ }^{14}$ H. R. Strauss, Plasma Phys. 22, 733 (1980). 